\title{
Optovibrometry: Tracking changes in the surface tension and viscosity of multicomponent droplets in real-time
}

\author{
Victoria C. Harrold and James S. Sharp ${ }^{1}$ \\ School of Physics and Astronomy, University of Nottingham, Nottingham, NG7 2RD, UK \\ ${ }_{1}$ Corresponding author, email:james.sharp@nottingham.ac.uk
}

\begin{abstract}
An instrument was developed for measuring real time changes in the surface tension and viscosity of multicomponent droplets of miscible liquids and other soft materials. Droplets containing glycerol and water were supported on superamphiphobic surfaces and vibrated by applying a short mechanical impulse. Laser light was refracted through the droplets and allowed to fall on the surface of a photodiode. Time dependent variations in the intensity measured by the photodiode during vibration were used to monitor the decay of the droplet oscillations. The frequencies and spectral widths of the droplet vibrational resonances were then obtained from Fourier transforms of these time dependent intensity signals. A recently developed model of droplet vibration was used along with these values and measurements of the drop dimensions to extract the surface tension and viscosity of the drops as they evaporated. Collection of data was automated and values of frequency, spectral width, drop size, surface tension and viscosity were obtained with a time resolution of three seconds over a period of thirty minutes. The values of surface tension and viscosity obtained were shown to be in good agreement with literature values obtained from bulk glycerol/water solutions; thus validating the technique for wider application to other soft matter systems.
\end{abstract}

\section{Introduction}

Droplet vibration has been studied for some time as a means of trying to extract physical properties such as the surface tension and viscosity of fluids [1-8]. Much of the early work in this field concentrated on the study of levitated droplets of single component liquids, whose properties could be extracted using theories based upon the theoretical models developed by Rayleigh [9] and Chandrasekhar [10]. These authors showed that the vibrational frequencies and damping coefficients associated with the vibration of a free liquid globe (essentially a levitated liquid drop) could be related independently to the surface tension and viscosity of the fluid using a simple set of equations. More recent studies have shown that a similar approach can also be used to extract the frequency dependent rheological properties of viscoelastic fluids [11].

Confirmation of these results came when the technology to levitate small liquid droplets became available in the form of acoustic [2] and magnetic levitation devices [3]. Much of the initial work in this area seems to have been motivated by the desire to perform containerless crystallisation studies on pure liquids in microgravity environments $[12,13]$. Later work turned to the use of this technique for extracting the physical properties of the drops and number of authors were able to demonstrate the viability of drop vibration as a means of extracting surface tension $[7,14]$ and viscosity $[1,7]$.

Other techniques are available that will allow the measurement of surface tension and/or viscosity of small volumes of simple liquids and multicomponent mixtures. Drop volume [15] and pendant drop [16] based techniques are among the more successful approaches for measuring surface tension, while piezorheology[17] and microrheology [18] based methods allow for the viscosity or viscoelastic properties of relatively small volumes of liquid to be determined. However, these types of technique very often provide a means of measuring only one of the required parameters.

Another method of extracting these properties is to vibrate substrate supported (sessile) or pendant drops. The ease with which a drop can be placed on a substrate, or suspended from a pipette and vibrated makes this approach more attractive and more accessible. There are, however, a number of challenges in interpreting the drop vibration data for substrate supported drops. Firstly, the presence of a substrate (or pipette) influences the drop shape and has an influence on the vibrational frequency $[5,6,8]$. Gravity can also play a role in changing the frequencies of vibration if the droplets exceed a critical size. This size is set by the capillary length of the fluid, $l_{c}$,- above which gravitational effects 
dominate the shape of the drop and below which surface tension tends to dominate $[5,6,8]$. In the surface tension dominated regime, sessile drops assume the shape of a spherical cap in attempt to minimise their total surface, while in the gravity dominated regime the drops become flattened.

The proximity of the substrate to the free surface of the drop also introduces additional energy dissipation mechanisms that are not present in levitated drops. These energy dissipation mechanisms affect the damping coefficients associated with the time dependent decay of the oscillations. The increased damping also causes an increase in the width of the peaks in the mechanical vibrational spectra of the drops [6]. The first of these energy dissipation mechanisms arises because velocity gradients within the droplet are much greater than those near the surface of an infinitely deep liquid bath. In a drop, the fluid velocities decay from the free surface value to zero near the substrate interface over distances that are comparable to the drop height. For shallow three phase contact angles these gradients can be very steep and can significantly increase the amount of energy dissipation and damping in the drop relative to the bulk fluid [6]. A second mechanism of energy dissipation arises when the three phase contact line at the liquid/air/substrate interface becomes depinned. In this case, motion of the contact line forces fluid to be dragged backwards and forwards across the substrate dissipating energy in a way that depends upon both drop size and shape [19].

One way to circumvent the difficulties associated with the presence of the substrate interface is to use non-wetting substrates such as superhydrophobic or superamphiphobic materials [20]. When drops are placed on these surfaces, the energetics of the interaction between the fluid and the substrate are such that the drops bead up and form spheres with three phase contact angles that are close to 180 degrees. In this way the drops assume shapes that are reminiscent of levitated drops and much of the physics of vibration can be described by using similar models.

McHale and coworkers [21] demonstrated the feasibility of this approach in studies of vibrating liquid marbles - liquid droplets stabilised by the adsorption of small particles on their surface. They showed that the vibrational frequencies of the liquid marbles could be described using expressions similar to those used for levitated droplets. However, the particles were found to dominate their properties, giving this particular approach limited application in the measurement of surface tension.

Recent studies of viscoelastic fluids supported on superamphiphobic substrates [22] have also shown that their vibrational response can be described using similar models to those used for levitated drops of the same materials [11]. These authors showed that measurements of the drop size, vibrational frequency and width of the vibrational peaks can be used to extract the frequency dependent shear storage and loss moduli of high molecular weight poly(acrylamide-co-acrylic acid) solutions in water [22].

Much of the work on drop vibration to date has concentrated on single liquids or on viscoelastic systems where the concentration of added solute doesn't change significantly with time. Here we motivate the idea that drop vibration can also be used to track the surface tension and viscosity of droplets supported on superamphiphobic substrates when the composition of the drops is changing with time. An automated combined light scattering and imaging technique is described for measuring the size, frequencies and spectral widths of vibrating drops of multicomponent fluids. These quantities are related to the surface tension and viscosity of the multicomponent fluid using a simplified version of a model developed for extracting the properties of viscoelastic droplets on the same substrates [22]. A series of experiments using drops containing different initial compositions of glycerol and water are used to test the validity of the vibration technique for studying the kinetics of changes in surface tension and viscosity as the droplets evaporate.

While the multicomponent drops studied here can be considered as soft matter systems in their own right, it is important to stress that a similar approach can be used to study other soft matter systems. As mentioned above, a similar technique has already been used by the present authors to study the vibrational properties of viscoelastic polymer solution droplets [22]. However, the application of this technique to the measurement of time dependent changes in the rheological properties of aggregating protein solutions, the clotting of blood droplets and in the monitoring the progress of gelation or selfhealing processes would be equally valid. The use of the simple system described here and the development of the experimental and theoretical protocols required to extract surface tension and 
rheological data enable us to validate this technique as a means of tracking material properties of soft systems in real time.

\section{Experimental}

Superamphiphobic substrates were prepared on glass surfaces as described previously [20,22]. Briefly, glass microscope slides were annealed in the flame of a parrafin candle until a black soot developed. The soot was then exposed to a combination of tetraethoxysilane (TEOS, Sigma-Aldrich, $98 \%$ ) and ammonia vapour (VWR, 28\%) for $72 \mathrm{~h}$ at room temperature. The resulting substrates were then annealed at $100{ }^{\circ} \mathrm{C}$ for $3 \mathrm{~h}$ to calcify the soot and form a fractal-like silica network on the surface. These substrates were then exposed to a second chemical vapour deposition of the semiflourinated silane, (tridecafluoro-1,1,2,2-tetrahydrooctyl)-1-trichlorosilane (Sigma, 97\%) for $3 \mathrm{~h}, 25$ mbar at room temperature, resulting in an advancing contact angle of $180 \pm 2^{\circ}$, a receding angle of $166 \pm 2^{\circ}$ and a roll-off angle of less than $2^{\circ}[22]$.

Glycerol and water were used as a model system for these experiments because they are miscible in all proportions and the viscosity of their mixtures varies over three orders of magnitude (1mPas-1Pas [23]). Microlitre droplets containing glycerol/water mixtures with compositions in the range 1-50 wt\% (0.008-0.44 volume fraction) were placed on the surface of the superamphiphobic substrates. Droplets were vibrated by applying a mechanical impulse from below using a SF-9324 mechanical wavedriver (Pasco Scientific, see figure 1a and 1b) that was driven using a USB-6211 data acquisition card (National Instruments) via a custom built amplifier. The small regions of contact between the droplets and the substrate were observed to remain pinned during drop vibration. However, vibrational waves were observed on the surface of the drops following the application of the mechanical impulse.

Laser light was refracted through the droplets and allowed to fall on the surface of a photodiode. During vibration, the refracted laser spot was observed to move across the surface of the photodiode; causing it to output a time dependent electrical signal similar to that shown in figure 1c. Two seconds of data were acquired from the photodiode at a rate of 1000 samples per second using the same data acquisition card used to generate the mechanical impulse. The resulting signal was Fourier transformed to obtain the mechanical vibrational spectrum of the droplet (see figure 1d) and the frequencies and spectral widths (full width at half maximum) of the vibrational modes of the drops were obtained from the dominant peaks (See inset, figure 1d). Both of these values were found to depend upon the size and composition of the drops for each vibrational mode. 

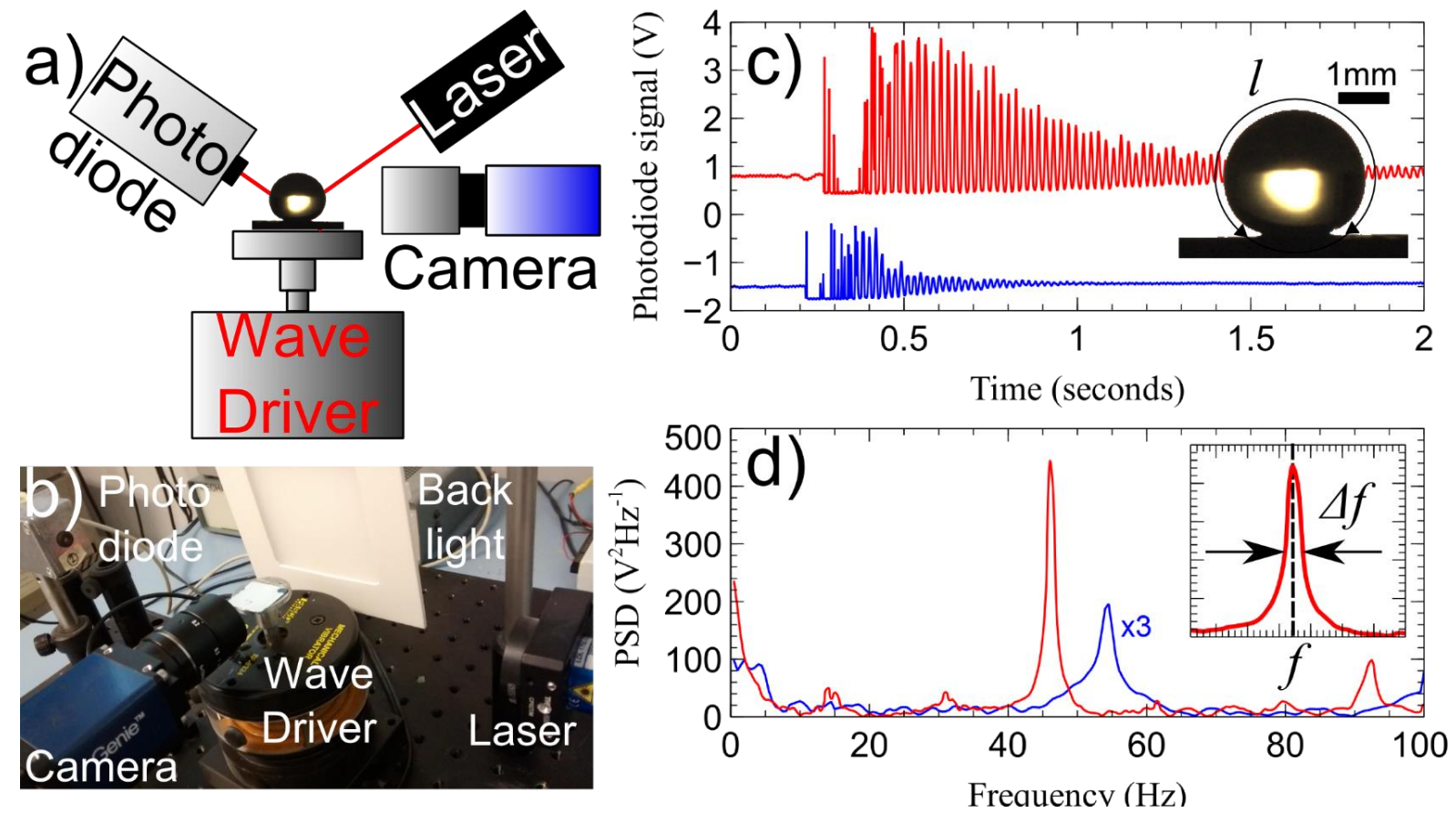

Figure 1. Light scattering apparatus for extracting the time dependent properties of multicomponent drops. Panels a) and b) show a schematic diagram and photograph of the setup used. Laser light is scattered through a drop supported on a superamphiphobic substrate and allowed to fall on the surface of a photodiode. Photographs of the backlit drop are used to measure the profile length of the drops as defined in the inset in panel c). The main panel in c) shows examples of the signals measured by the photodiode for droplets of $5 \mathrm{wt} \%$ (red) and $50 \mathrm{wt} \%$ (blue) glycerol of similar size $(\sim 4.6 \mu \mathrm{l}$ volume $)$ following the application of a mechanical impulse at approximately 0.2 seconds. Panel d) shows the corresponding Fourier transforms of the data in panel c). The inset in this panel shows how the frequency, $f$, and width, $\Delta f$ are defined. 
Images of backlit drops were captured prior to vibration using a Genie HC640 camera (Teledyne Dalsa, see figure 1b). These were used to measure the profile length, $l$, of the drops as shown in the inset in figure 1c. The drops studied had radii smaller than the capillary length of the fluids and as such had shapes that are close to that of a perfect sphere when deposited on the superamphiphobic substrates with contact angles close to $180^{\circ}$. As a result, the profile length could have been approximated to the circumference of the drop i.e. $l=2 \pi R$. However, the use of the measured $l$ value, rather than the circumference allowed deviations in the contact angle to occur away from $180^{\circ}$ without them significantly influencing the data analysis. This reduced the uncertainties associated with the measured values of the drop size/profile length.

Software written in LabView (National Instruments) was used to automate the collection of image, drop size and vibration data- including the time dependent photodiode signals and corresponding Fourier transforms. The same software was also used to analyse the data and calculate the surface tension and viscosity of the glycerol/water solutions as the drops evaporated. The minimum time resolution of the measurement and analysis process was three seconds and allowed changes in surface tension and viscosity to be tracked in real time. All measurements were performed at room temperature which was maintained at $20+/-2{ }^{\circ} \mathrm{C}$. The relative humidity in the room was measured to be around $50+/-5 \%$ during the measurements.

\section{Results and Discussion}

Figure 2 shows examples of data collected for four drops of similar size that had different initial glycerol/water compositions. As the droplets evaporated, the size (profile length) of the drops was observed to decrease and both the frequency and width of the lowest vibrational mode increased with time. The increases in the latter two parameters occurred as a result of the shrinking dimensions of the drops and due to changes in the surface tension and viscosity. Smaller liquid drops have higher vibrational frequencies and experience more damping $[6,8]$, but at the same time, the loss of one or more of the droplet components is expected to cause changes in the material properties of the mixture. 

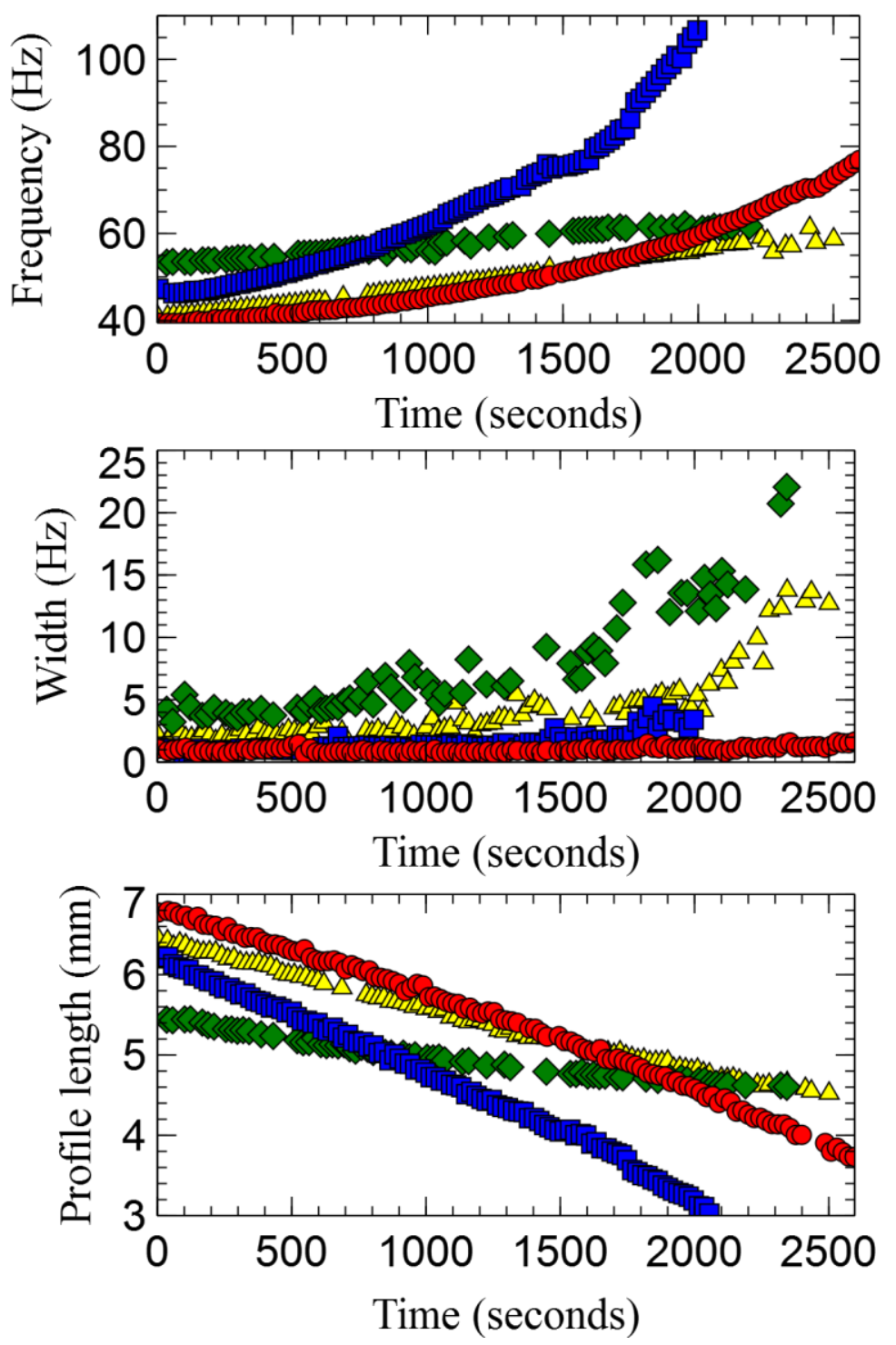

Figure 2. Time dependent changes in the properties of glycerol/water droplets. The panels show how the vibrational frequency (top), spectral width (middle) and profile length of droplets varies with time. Data are shown for four droplets of similar size having initial compositions of $1 \mathrm{wt} \%$ (red circles), $5 \mathrm{wt} \%$ (blue squares), $25 \mathrm{wt} \%$ (yellow triangles) and $50 \mathrm{wt} \%$ (green diamonds) of glycerol. The initial compositions correspond to glycerol volume fractions of $0.008,0.04,0.209$ and 0.44 respectively.

When interpreting the data for the binary mixtures used in these experiments it was assumed that the dominant source of mass loss occurred as the result of evaporation of water. This assumption was supported by the observation that pure glycerol droplets did not evaporate on the time scales of the experiments ( 2000s). However, microlitre droplets of pure water were observed to undergo significant mass loss as a result of evaporation over similar time scales.

Decoupling the effects of the changing size of the drops from changes in surface tension and viscosity requires some method of interpreting the vibrational frequencies, $f$, and widths, $\Delta f$, measured during the experiment. There have been numerous studies which have developed theories and models that have attempted to relate these quantities with a great deal of success for levitated $[9,10]$, pendant $[8,12]$ and sessile [4-6,12,13,24] drops. Harrold et al. [22] recently showed that the generalised viscoelastic properties of a vibrating drop supported on a superamphiphobic substrate could be described by considering standing wave states on the surface of the drops. These authors used the dispersion relation for capillary waves on the surface of a viscoelastic fluid first introduced by Pleiner, Harden and Pincus [25]; 


$$
4 \pi^{2} \rho(f+i \Delta f)^{2}=\frac{4 i(f+i \Delta f)\left(G^{\prime \prime}(f)-i G^{\prime}(f) k^{2}\right)}{f}+\gamma k^{3}
$$

where $\gamma$ is the surface tension, $\rho$ is the density and $G^{\prime}$ and $G$ "' are the shear storage and loss moduli of the droplet material respectively.

This equation was used in conjunction with a condition that a half integer number of wavelengths fit around the profile length of the drops at resonance i.e. $n \frac{\lambda}{2}=l$, which gave values for the allowed wavevector magnitudes of $k=\frac{2 \pi}{\lambda}=\frac{n \pi}{l}$, where $n$ is a mode number. Inserting this result for $k$ into equation 1 and equating real and imaginary parts gave expressions for $G$ ' and $G$ " of the form;

$$
\begin{aligned}
G^{\prime} & =\frac{\rho f^{2} l^{2}}{n^{2}}\left(1-\frac{\pi \gamma n^{3}}{4 \rho l^{3}\left(\Delta f^{2}+f^{2}\right)}\right) \\
G^{\prime \prime} & =\frac{\rho f \Delta f l^{2}}{n^{2}}\left(1+\frac{\pi \gamma n^{3}}{4 \rho l^{3}\left(\Delta f^{2}+f^{2}\right)}\right)
\end{aligned}
$$

These expressions were shown to give values for $G^{\prime}$ and $G$ "' that agreed well with the frequency dependent rheological properties of viscoelastic drops of poly(acrylamide-co-acrylic acid) in water with dimensions below the capillary length of the fluid [22].

For the simple liquids studied here, the above equations can be simplified further by setting $G^{\prime}=0$ (no elastic component) and noting that the viscosity of the drops is given by $\eta=\frac{G^{\prime \prime}}{2 \pi f}$. This gives expressions for the surface tension and viscosity of the form;

$$
\gamma=\frac{4 \rho l^{3}}{\pi n^{3}}\left(\Delta f^{2}+f^{2}\right)
$$

and

$$
\eta=\frac{\rho \Delta f l^{2}}{\pi n^{2}}
$$

The functional forms of the expressions given in equations 3 and 4 compare favourably with those obtained for the corresponding equations for levitated drops $[3,9,10]$. However, the expressions derived here are modified slightly. This is caused by the fact that a vibrational node must exist in the contact region with the substrate if the droplets remain pinned during vibration- a condition which is not imposed on levitated drops [11]. This additional constraint has the effect of changing the prefactor in the relevant equations above when compared to the equivalent expressions for levitated drops. Another difference between the equations derived above and those reported for levitated droplets is the addition of the width dependence in the surface tension equation. For inviscid fluids, this term is often neglected as damping is relatively weak and has little effect on the vibrational frequencies of the droplets and the corresponding surface tension values. However, for the fluids studied here the viscosity could potentially vary over three orders of magnitude. Damping of the droplets will therefore have a significant effect upon their motion. This is particularly true for the higher glycerol concentrations where the increased damping is expected to influence the vibrational frequencies of the droplets. The addition of the width dependence in equation 3 takes these effects into account and compensates for the effects of shifts in vibrational frequency caused by damping of a mechanically vibrating system [26]. 

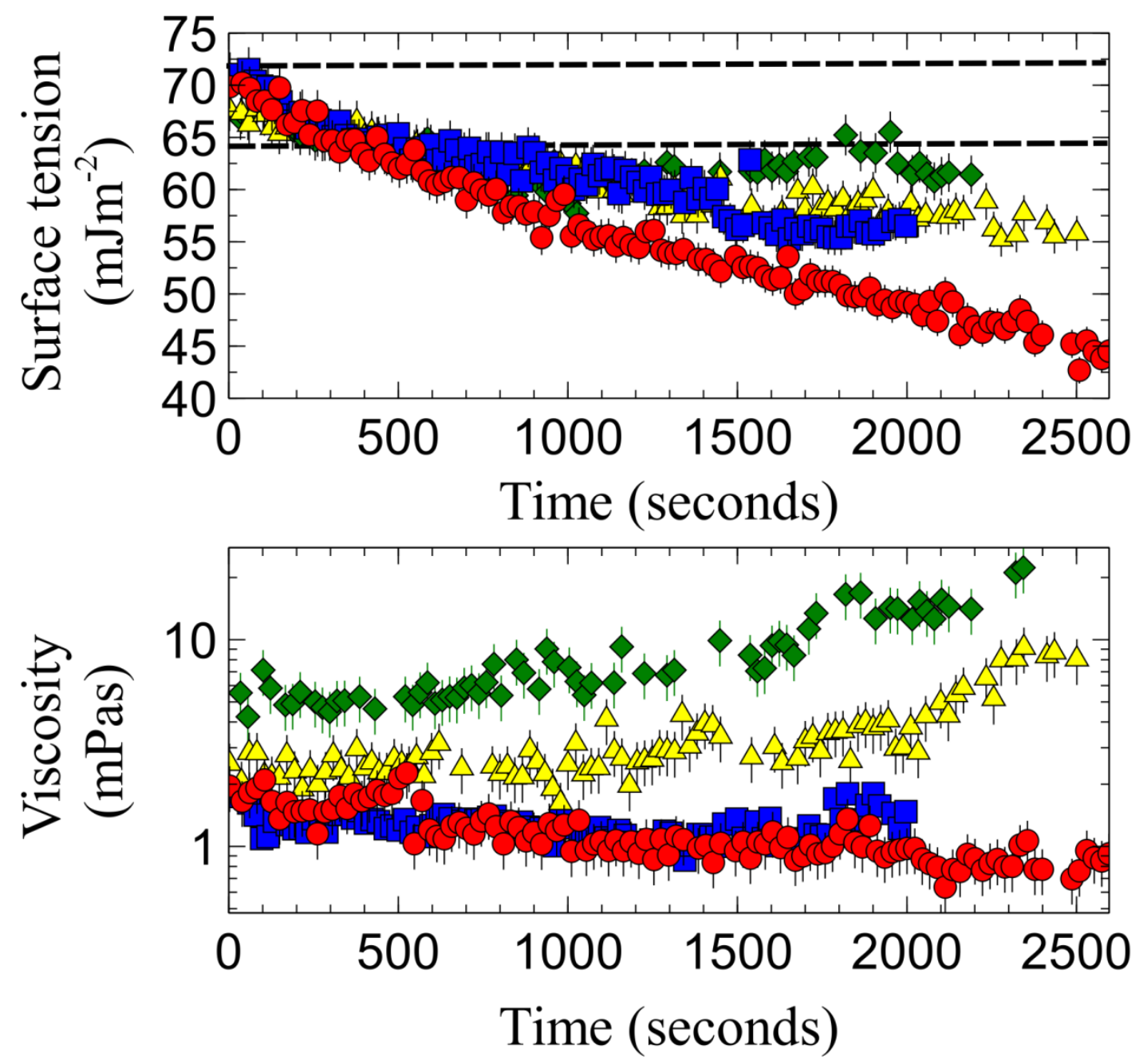

Figure 3. Time dependence of the surface tension (top panel) and viscosity (bottom panel) of evaporating glycerol/water solutions. Data are shown for four droplets of similar size having initial compositions of $1 \mathrm{wt} \%$ (red circles), $5 \mathrm{wt} \%$ (blue squares), $25 \mathrm{wt} \%$ (yellow triangles) and $50 \mathrm{wt} \%$ (green diamonds) of glycerol. The initial compositions correspond to glycerol volume fractions of $0.008,0.04,0.209$ and 0.44 respectively. The dashed horizontal lines in the top panel correspond to the surface tensions of pure water and pure glycerol respectively

Figure 3 shows the time dependent variation of the surface tension and viscosity for the droplet data shown in figure 2. The measured values of $f, \Delta f$ and $l$ in figure 2 were used to calculate $\gamma$ and $\eta$ at each time point using equations 3 and 4 . A value of $n=2$ was used for the lowest mode of vibration as has been discussed previously for vibrating drops with pinned contact regions $[5,6,8,22,27]$.

The changing composition of the drops meant that their density varied somewhere in the range between $998 \mathrm{kgm}^{-3}$ (pure water,[23]) and $1260 \mathrm{kgm}^{-3}$ (pure glycerol,[23]) during the experiments. At each time point the volume of the drop was calculated using the equation, $V(t)=\frac{l^{3}}{6 \pi^{2}}$. The volume fraction of glycerol was then calculated by assuming that mass loss occurred purely as the result of the evaporation of water. This gave an equation for the volume fraction of glycerol in the drop, $\phi(t)$, such that

$$
\phi(t)=\phi_{o} \frac{V_{0}}{V(t)}
$$


where $\phi_{o}$ and $V_{o}$ are the initial glycerol volume fraction and volume of the drop respectively. Values of the density of the drops were then calculated using a fit to data obtained by Sheely [23] for bulk glycerol/water solutions (see supplementary information). This fit gave the result that the density of the drops varied according to the equation $\rho=988+270 \phi(t)$. Insertion of the volume fraction dependence of the density in equations 3 and 4 allowed the correct surface tension and viscosity values to be extracted for the evaporating droplets at each time point.

The top panel in figure 3 shows that the surface tension of the drops varies with time in a manner that is almost independent of the drops' initial composition. Moreover, the surface tension varies over a range of values that is larger than that expected for glycerol/water solutions. If the changes in surface tension were caused purely by a change in composition of the drops then the surface tension values should vary in a range somewhere between that of pure water $\left(72 \mathrm{mJm}^{-2}\right.$ [28]) and pure glycerol (63 $\left.\mathrm{mJm}^{-2}[28]\right)$. The final values of the surface tension obtained from these experiments were as low as $\sim 40 \mathrm{mJm}^{-2}$. Given that the drop vibration technique has already been shown to be a viable method of determining surface tension $[7,8,14]$, this would seem to suggest that some contamination of the surface of the drops is occurring during the experiments. The fact that the kinetics associated with the changes in surface tension are similar for all the droplets studied regardless of their initial compositions is also consistent with the presence of surface contamination.

Contamination of the drop surface could have occurred either as a result of contact with the substrate or as a result of deposition of airborne contaminants. There was no visible evidence of surface contamination of the surface by soot particles from the substrate. However, submicron sized fragments of soot, TEOS or the fluorinated silane that was used to coat the candle soot could potentially have migrated across the surface of the droplets during the experiments. A series of simple tests were performed to determine whether this type of surface contamination was occurring. These simple tests were performed by filling a clean $20 \mathrm{~cm}$ diameter glass beaker with water and gently dusting the surface with talc. Fine syringe needles were either dipped in the silane of interest, or used to mechanically scrape some of the candle soot residue off the surface of the substrates being used. These syringe needles were then gently pushed through the talc coated water surface at the centre of the glass beaker.

In the case of both TEOS and the fluorinated silane, a hole formed in the talc layer and was observed to grow to the diameter of the glass beaker in less than one second. This gives a lower bound for the spreading rate of these molecules of around $10 \mathrm{cms}^{-1}$. Spreading of TEOS or the fluorinated silane across the surface of the drops was therefore not responsible for the surface contamination observed in the drop vibration experiments. The time scale associated with the contamination of the microlitre drops studied would be $\sim 0.03$ seconds for a drop with a profile length of $\sim 6 \mathrm{~mm}$. This is at least four orders of magnitude smaller than the timescale associated with the surface tension changes shown in figure 3 and indicates that the candle soot substrates contain no free surfactant like molecules that could contaminate the surface. However, there was still a possibility that particles of soot were able to break off from the surface of the substrate and contaminate the drop surface. A similar test, repeated with mechanically scraped soot from the substrate showed no discernible change in the talc layer over a period of 2000s i.e. no hole was observed to form. While not entirely perfect, these simple tests suggest that the superamphiphobic substrates used in this work were mechanically and chemically stable on the timescales associated with the experiments and that they were not responsible for contaminating the drop surface. This would suggest that airborne contamination of the droplets surfaces is a more likely candidate for the surface tension changes observed in figure 3.

Henderson and Miles [29] demonstrated that a clean water surface can become contaminated with low surface tension impurities such as organic deposits from the air on time scales of $\sim 5$ mins $(300$ seconds). Figure 3 shows that the surface tension of the glycerol/water drops changes on time scales of hundreds of seconds and falls below the surface tension of glycerol after $\sim 300$ seconds for all the compositions studied. Both water and glycerol have relatively high surface tensions compared to organic materials, which typically have $\gamma \sim 40-50 \mathrm{mJm}^{-2}$ [30]. It is therefore not unreasonable that contamination could occur as a result of the preferential adsorption of species with lower surface tension values on the drop surface. The fact that the surface tensions of the drops approached values similar to those associated with organic materials lends additional support to this argument.

The surface tension data in figure 3 was plotted as a function of the instantaneous volume fraction of glycerol in the drop as shown in the inset in figure 4 . The initial values of the surface tension were 
found to agree with independent measurements of fresh solutions obtained using the pendant drop technique (white squares) before significant exposure to the air had occurred [16]. The agreement between the surface tension values at $\mathrm{t}=0$ and the pendant drop measurements gives us confidence that the technique is capable of measuring the correct surface tension values for the initially uncontaminated drops. However, as time progresses and the exposure to air increases, the surface tension values obtained during the drop vibration experiments deviate from those obtained for clean solutions.

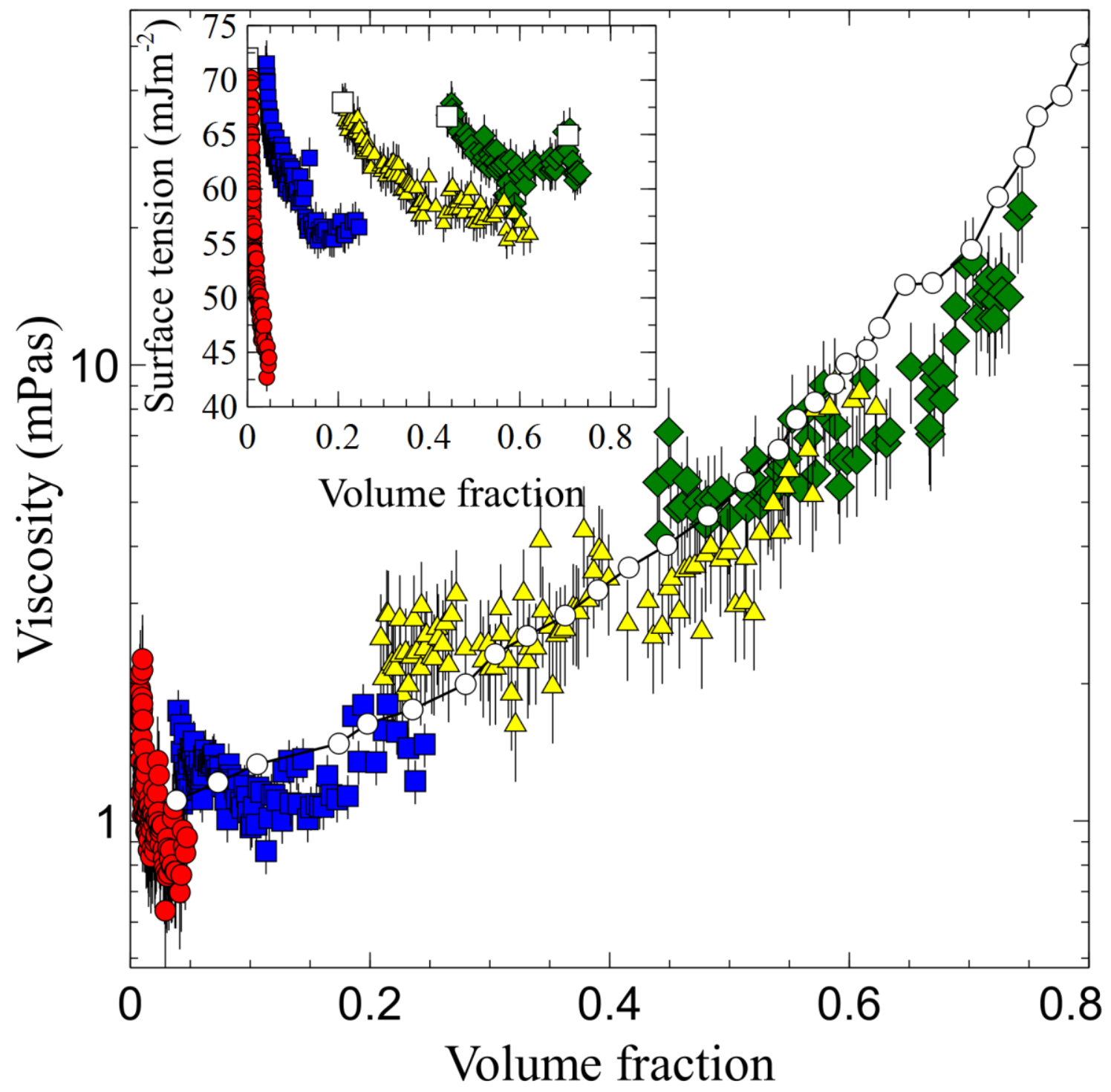

Figure 4. Composition dependence of the properties of glycerol/water solutions. The main panel shows the variation of the viscosity of glycerol/water solutions obtained during the evaporation experiments shown in figures 2 and 3 as a function of the glycerol volume fraction. Data are shown for droplets having initial compositions of $1 \mathrm{wt} \%$ (red circles), $5 \mathrm{wt} \%$ (blue squares), $25 \mathrm{wt} \%$ (yellow triangles) and 50wt\% (green diamonds) of glycerol. The initial compositions correspond to glycerol volume fractions of $0.008,0.04,0.209$ and 0.44 respectively. The white circles show results for the bulk viscosity of glycerol/water solutions obtained by Sheely [23] at $20^{\circ} \mathrm{C}$. The inset shows a plot of the surface tension as a function of composition for the same droplets. The white squares in this plot are the results of pendant drop measurements of the surface tension obtained from clean solutions, prior to contamination from the air (see text).

Figure 3 also shows how the viscosity of the droplets varies as a function of time. Unlike surface tension, viscosity is a bulk property and should therefore be unaffected by the surface contamination effects discussed above. The plots in figure 3 show that the initial viscosity obtained using equation 4 
increased with increasing glycerol composition of the drops. Moreover, for the two highest initial glycerol/water compositions studied (25 and $50 \mathrm{wt} \%$ glycerol), the viscosity was observed to increase further as the droplets evaporated and the glycerol composition increased -the range of values obtained being bounded by the viscosities of pure water (1mPas) and glycerol (1Pas).

To determine whether the values of viscosity shown in figure 3 are consistent with values expected for the composition of the drops, the same data was plotted in the main panel of figure 4 against the corresponding instantaneous volume fraction of glycerol in the drops. Values obtained from Sheely [23] for bulk glycerol/water solutions at a temperature of $20^{\circ} \mathrm{C}$ are also shown for comparison (white squares). The droplet data is shown to agree with the bulk solution viscosity data within the limits of experimental uncertainty. Any small deviations of the drop vibration data away from the bulk data are believed to occur as the result of small temperature variations. Such a drift in temperature is likely to have caused the slight downward drift in viscosity with time observed for the two lowest initial compositions ( 0 and $5 \mathrm{wt} \%$ ) shown in figure 3 . The fact that the viscosities obtained for these two compositions are consistent with literature values, within the limits of experimental uncertainty (as shown in figure 4), suggests that these data are representative of the glycerol water mixtures being studied. However, these data suggest that the changes in viscosity measured using the vibration techniques described above are more susceptible to variations in temperature for low glycerol concentrations - pointing to the need for greater temperature control.

Despite the small temperature related effects described above, the level of agreement observed between literature values and the volume fraction dependence of the viscosity shown in figure 4 is encouraging. It demonstrates that the model described above captures the essential physics of drop vibration. It also demonstrates the validity of the assumption that volume changes in the drops are caused by the evaporation of water.

The automated approach to drop vibration described above provides an inexpensive method of simultaneously tracking changes in the surface tension and viscosity of microlitre liquid droplets regardless of whether they occur as the result of surface contamination effects or simply as a result of changes in composition of the drops. One limitation of the technique is that the composition dependence of the density is required to obtain the surface tension and dynamic viscosity of the solutions. However, the ratio of these quantities to the density can be obtained directly from the measurements. In the case of viscosity, this ratio is referred to as the kinematic viscosity.

A further limitation of this approach is that the size of the droplet must be smaller than the capillary length of the fluid for the assumptions made in deriving the dispersion relation in equation 1 to hold. However, the requirement for a small sample volume is likely to be of benefit for studying expensive samples and samples where volumes are low (such as blood samples).

Caution should also be exercised when applying the results of the simple model derived here to drop vibration studies. The dispersion relation of Pleiner, Harden and Pincus [25] was derived for capillary waves on the surface of an infinitely deep (viscoelastic) fluid. Application of this dispersion relation to curved droplet interfaces is therefore questionable. In the limit where the amplitude of surface deflections of the drops becomes small compared to their size/radius $(<0.1 R)$ this approximation has been shown to hold. However, for larger amplitude vibrations and in cases where the contact region becomes depinned during vibration, the validity of this approximation becomes significantly weaker [22]. In such cases, more rigorous theories which consider a full expansion of the spherical harmonics associated with surface fluctuations of the droplets should be applied [13,24,31-33].

\section{Conclusion}

Automated light scattering measurements can be used to probe changes in the surface tension and viscosity of multicomponent microlitre droplets simultaneously and in real-time. The values obtained from drop vibration measurements of evaporating glycerol/water droplets were found to be in good agreement with data obtained from bulk glycerol/water solutions. The simple apparatus described here is inexpensive and easy to implement. Its portability lends it to applications in a broad range of settings including microfluidics devices, ambient, high pressure and ultra-high vacuum (e.g. ionic liquids) environments. Moreover, the ease with which this technique can be implemented potentially lends itself to the study of a range of soft matter systems including (but not limited to) aggregating 
protein solutions, blood droplet rheology, gelation reactions, self-healing materials, viscoelastic polymer solutions and liquid crystals.

\section{Acknowledgements}

The authors thank Doris Vollmer and Maxime Paven (Max Planck Institute for Polymer Research, Mainz) for providing the superamphiphobic substrates used in this work. JSS also thanks the LeverHulme Trust for providing funding under a Research Project Grant (RPG-2012-702).

\section{References}

[1] I. Egry, G. Lohöfer, I. Seyhan, S. Schneider and B. Feuerbacher, "Viscosity of eutectic

$\mathrm{Pd}_{78} \mathrm{Cu}_{6} \mathrm{Si}_{16}$ measured by the oscillating drop technique in microgravity", Appl. Phys. Lett., 73(4), 462463, (1998).

[2] C.L. Shen, W.J. Xie and B. Wei, "Parametrically excited sectorial oscillation of liquid drops floating in ultrasound", Phys. Rev. E., 81, 046305, (2010).

[3] R.J.A. Hill and L. Eaves, "Vibrations of a diamagnetically levitated water droplet", Phys. Rev. B, 81, 056312, (2010).

[4] X. Noblin, A. Buguin and F. Brochard-Wyart, "Vibrations of sessile drops”, Eur. Phys. J.: Spec. Top., 166, 7-10, (2009).

[5] J.S. Sharp, D.J. Farmer and J. Kelly, "Contact angle dependence of the resonant frequency of sessile water droplets", Langmuir, 27, 9367-9371, (2011).

[6] J.S. Sharp, "Resonant properties of sessile droplets; contact angle dependence of the resonant frequency and width in glycerol/water mixtures", Soft Matter, 8, 399-407, (2012).

[7] W. Meier, G. Gruene, A. Meyboon and K.P. Hofmann, "Surface tension and viscosity of surfactant from the resonance of an oscillating drop", Eur. Biophys. J., 29, 113-124, (2000).

[8] R.H. Temperton, M.I. Smith and J.S. Sharp, "Mechanical vibrations of pendant liquid droplets", Eur. Phys. J. E, 38, 79-86, (2015).

[9] R.W.S. Rayleigh, "On the capillary phenomena of jets", Proc. R. Soc. London, 29, 71-97, (1879).

[10] S. Chandrasekhar, "Oscillations of a viscous liquid globe", Proc. London Math. Soc., 3(9), 141149, (1959).

[11] R.H. Temperton, R.J.A. Hill and J.S. Sharp, "Mechanical vibrations of magnetically levitated viscoelastic droplets", Soft Matter, 10, 5375-5379, (2014).

[12] E.D. Wilkes and O.A. Basaran,"Forced oscillations of pendant (sessile) drops" Phys. Fluids, 9(6), 1512-1528, (1997)

[13] M. Strani and F. Sabetta,"Free vibrations of a drop in partial contact with a solid support" J. Fluid Mech., 141, 233-247, (1984).

[14] I. Egry, H. Giffard and S. Schneider, "The oscillating drop technique revisited", Meas. Sci. Technol., 16, 426-431, (2005).

[15] R. Miller, A. Hofmann, R. Hartmann, K-H. Schano and A. Halbig, "Measuring dynamic surface and interfacial tensions", Advanced Materials, 4(5), 370-374, (1992).

[16] O.I. Del Rio and A.W. Neumann, "Axisymmetric drop shape analysis: computational methods for the measurement of interfacial properties from the shape and dimensions of pendant and sessile drops", J. Colloid Int. Sci., 196, 136-147, (1997).

[17] M. Roth, M. D’Acunzi, D. Vollmer and G. K. Auernhammer, "Viscoelastic rheology of colloid-liquid crystal composites", J. Chem. Phys., 132, 124702, (2010).

[18] T.G. Mason and D.A. Weitz, "Optical measurements of frequency dependent linear viscoelastic moduli of complex fluids", Phys. Rev. Lett., 74, 1250, (1955).

[19] X. Noblin, A. Buguin and F. Brochard-Wyart,"Vibrated sessile drops: Transition between pinned and mobile contact line oscillations", Eur. Phys. J. E., 14, 395-404, (2004).

[20] X. Deng, L. Mammen, H-J. Butt and D. Vollmer, "Candle soot as a template for a transparent robust superamphiphobic coating", Science, 335, 67-70, (2012).

[21] G. McHale, S.J. Elliot, M.I. Newton, D.L. Herbertson and K. Esmer, "Levitation-free vibrated droplets: resonant oscillations of liquid marbles", Langmuir, 25, 529-533, (2009).

[22] V.C. Harrold, M. Paven, D. Vollmer and J.S.Sharp, "Rheological properties of viscoelastic drops on superamphiphobic substrates", Langmuir, 32, 4071-4076, (2016).

[23] M.L. Sheely, "Glycerol viscosity tables", Ind. Eng. Chem., 24(9), 1060-1064, (1932).

[24] J.B. Bostwick and P.H. Steen, "Capillary oscillations of a constrained liquid drop", Phys. Fluids, 21, 032108, (2009).

[25] H. Pleiner, J.L. Harden and P. Pincus, "Surface-modes on a viscoelastic medium", EuroPhys. Lett., 7(5), 383-387, (1988). 
[26] R.D. Knight, "Physics for Scientists and Engineers",2 ${ }^{\text {nd }}$ Ed., Pearson Addison-Wesley (San Francisco), 429, (2008)

[27] R.H. Temperton and J.S. Sharp, "Vibrational modes of elongated sessile droplets", Langmuir, 29, 4737-4742, (2013).

[28] C.S. Milner and N.N. Dalton Eds., "Glycerol", Reinhold (New York), 326, (1953).

[29] D.M. Henderson and J.W. Miles, "Surface-wave damping in a circular cylinder with a fixed contact line", J. Fluid Mech., 275, 285-299, (1994).

[30] R.C. Weast (Editor), "Handbook of Chemistry and Physics", 69th ed. , CRC Press (London), (1989).

[31] D.V. Lyubimov, T.P. Lyubimova and S.V. Shklyaev, "Behaviour of a drop on an oscillating solid plate", Phys. Fluids, 18, 012101, (2006).

[32] S. Ramalingam, D. Ramkrishna and O.A. Basaran, "Free vibrations of a spherical drop constrained at an azimuth", Phys. Fluids, 24, 082102, (2012).

[33] A. Prosperetti, "Linear oscillations of constrained drops, bubbles and plane liquid surfaces", Phys. Fluids, 24, 032109, (2012). 